\title{
Double disadvantage: a case control study on health-related quality of life in children with sickle cell disease
}

\author{
Channa T Hijmans ${ }^{1,2 *}$, Karin Fijnvandraat ${ }^{2}$, Jaap Oosterlaan ${ }^{3}$, Harriët Heijboer ${ }^{2}$, Marjolein Peters²,
} Martha A Grootenhuis ${ }^{1}$

\begin{abstract}
Background: Low health-related quality of life (HRQoL) of children with sickle cell disease (SCD) may be associated with consequences of the disease, or with the low socio-economic status (SES) of this patient population. The aim of this study was to investigate the HRQOL of children with SCD, controlling for SES by comparing them to healthy siblings (matched for age and gender), and to a Dutch norm population.

Methods: The HRQoL of 40 children with homozygous SCD and 36 healthy siblings was evaluated by the KIDSCREEN-52. This self-report questionnaire assesses ten domains of HRQoL. Differences between children with SCD and healthy siblings were analyzed using linear mixed models. One-sample t-tests were used to analyze differences with the Dutch norm population. Furthermore, the proportion of children with SCD with impaired HRQOL was evaluated.

Results: In general, the HRQOL of children with SCD appeared comparable to the HRQoL of healthy siblings, while children with SCD had worse HRQoL than the Dutch norm population on five domains (Physical Well-being, Moods \& Emotions, Autonomy, Parent Relation, and Financial Resources). Healthy siblings had worse HRQoL than the Dutch norm population on three domains (Moods \& Emotions, Parent Relation, and Financial Resources). More than one in three children with SCD and healthy siblings had impaired HRQoL on several domains.

Conclusion: These findings imply that reduced HRQoL in children with SCD is mainly related to the low SES of this patient population, with the exception of disease specific effects on the physical and autonomy domain. We conclude that children with SCD are especially vulnerable compared to other patient populations, and have special health care needs.
\end{abstract}

\section{Background}

Sickle cell disease (SCD) is a hereditary red blood cell disorder that occurs predominantly in people of African ancestry [1]. SCD is becoming one of the most common genetic disorders in children in Western Europe, due to demographic changes [2]. In the Netherlands, an estimated number of 1000 children, originating from Surinam and Central Africa, have SCD. The disease is characterized by chronic haemolytic anaemia and vascular occlusion, causing recurrent painful episodes,

\footnotetext{
* Correspondence: c.t.hijmans@amc.nl

'Psychosocial Department, Emma Children's Hospital, Academic Medical

Center, P.O. Box 304, 1100 VC Amsterdam, The Netherlands

Full list of author information is available at the end of the article
}

irreversible organ damage, and neurocognitive deficits. Besides the medical consequences, most families with a child with SCD have to cope with financial and social problems, as the majority belongs to immigrant communities with a low socio-economic status (SES) and is single parented [3]. Nevertheless, the differential impact of low SES and the disease specific consequences of SCD on health-related quality of life (HRQoL) in children is not well known.

Quality of Life (QoL) is defined as an individual's perception of one's position in life in the context of culture and value systems, as well as in relation to one's goals, expectations, standards and concerns. HRQoL is defined as QoL in which a dimension of personal judgement of 
one's health and disease is added [4]. In the case of children, HRQoL is also influenced by factors such as the ability to participate in peer groups and the ability to keep up with developmental activities. Difficulties in measuring HRQoL in children include a lack of consensus on suitable (cross-cultural), self-report instruments and the need for different instruments in different age groups. Recently, the KIDSCREEN-52 was developed in Europe as a generic, cross-national HRQoL questionnaire that evaluates HRQoL regardless of whether children are in good health or suffer from a chronic medical condition [5]. This self-report questionnaire evaluates three components of children's well-being, in line with the definition of HRQoL of the World Health Organization: the physical, psychological, and social component [4].

Previous research has evaluated psychological and social problems in children with SCD. Although findings were inconclusive, internalizing problems (such as anxiety and depression), social problems, and feelings of low self-esteem are commonly reported [6-10]. However, the measurement of HRQoL, encompassing all three components of children's well-being, is still in its infancy in children with SCD [11]. For example, compared to over 400 studies on HRQoL in children with cancer, research on HRQoL of children with SCD is just scratching the surface [12]. The relatively few studies that have been performed revealed that HRQoL of children with SCD is generally poor [13-18].

However, these studies have several limitations. First, some investigators solely relied on parental ratings of HRQoL [14,15], while self report has been described as the ideal when measuring HRQoL [11]. Second, almost all previous studies have been performed in the United States of America. As the healthcare system and the origins of the patient population in the USA differ from Western Europe, findings from these studies cannot be generalized to the European population of children with SCD. Although the first study on HRQoL of children with SCD was performed at our own institution and showed lower HRQoL on several domains [13], this study did not yet consider the impact of low socio-economic status (SES) on HRQoL. Subsequent studies that did specifically address the role of low SES yielded conflicting results, which can be ascribed to isolated concepts of SES that were used (e.g. parental work status, educational level, neighbourhood socioeconomic distress, or family income) [14-17]. SES is defined as a total measure of a person's work experience and of an individual's or family's economic and social position relative to others, based on income, education, and occupation. A more comprehensive approach to grasp all these economic and sociological aspects of SES could be to compare children with SCD to a control group of healthy siblings with the same background. So far, no studies on HRQoL of children with SCD have included healthy siblings as controls. Instead, most researchers compared children with SCD to (non-White) population norms or to a control group with a different ethnicity or income level.

The aim of the current study was to examine whether reduced HRQoL in children with SCD is related to consequences of the disease or to the low SES of most patients. Therefore, we investigated self-reported HRQoL of children with SCD compared to (1) healthy siblings (who are comparable in age, gender, ethnicity and SES) and (2) a Dutch norm population. We hypothesized that children with SCD would have a lower HRQoL than healthy siblings with the same background, suggesting reduced HRQoL to be related to consequences of the disease. Alternatively, if children with SCD and healthy siblings would have comparable HRQoL, but children with SCD would have a lower HRQoL than the Dutch norm population, this would suggest that reduced HRQoL is related to low SES.

\section{Methods \\ Participants}

Forty children with SCD and 36 healthy siblings aged 6 to 18 years participated in the study. A total of 46 children were randomly selected from the children receiving treatment for a severe form of SCD (HbSS or HbS- $\beta 0$ thalassemia) at the Comprehensive Sickle Cell Care Center of the Emma Children's Hospital, Academic Medical Center in Amsterdam. From these 46 children, $40(87 \%)$ participated (6 declined). Most of them had an HbSS genotype $(n=36,90 \%)$, the other four $(10 \%)$ had an HbS- $\beta 0$-genotype. The clinical condition of all children with SCD was stable at the time the HRQoL evaluation took place.

After recruitment of participants with SCD, healthy siblings of the group of participants were recruited. Fifty-seven healthy (full or half) siblings were invited to participate. From these, 36 (63\%) participated. Siblings were matched for age and gender one by one to participants. As no sibling match was available for all participating children with SCD, we recruited nineteen healthy siblings $(50 \%)$ in families from non-participating children with SCD receiving care at our hospital. These healthy siblings had similar demographic characteristics and were matched for age and gender one by one to participants as well. Inclusion took place between October 2007 and October 2008.

\section{Questionnaire}

HRQoL was evaluated by the KIDSCREEN-52, a generic self-report questionnaire that uses questions derived from focus groups of children and adolescents across 
Europe. It is applicable for both healthy and chronically ill children and adolescents aged between 8 and 18 years. The KIDSCREEN-52 consists of 52 items assessing ten domains of HRQoL during the previous week: 'Physical Well-being', 'Psychological Well-being', 'Moods \& Emotions', 'Self-Perception', 'Autonomy', 'Parent Relation \& Home Life', 'Financial Resources', 'Social Support \& Peers', 'School Environment' and 'Bullying'. Items are scored on a five-point scale. Within each domain, item scores are summed and transformed to a $\mathrm{T}$ value. Children in the European norm population have a mean score of 50 with a standard deviation (SD) of 10, with higher values indicating better HRQoL. This instrument is validated in a Dutch population of 1960 children, with an age distribution of 8 to 11 years $(\mathrm{n}=641)$ and 12 to 18 years $(\mathrm{n}=1270)$. Means of the Dutch norm population vary across domains, but are generally 2-3 points higher than in the European norm population. The instrument has satisfactory reliability and validity and good internal consistency [19].

\section{Procedure}

The medical ethics committee of the Academic Medical Center of Amsterdam approved the study protocol. Written informed consent was obtained from parents and from children aged twelve years and older. Children were invited to visit our outpatient clinic where the KIDSCREEN-52 was administered. Questions were read aloud by an interviewer for children with lower reading capabilities. Young participants received cognitive debriefing to ascertain they understood the questions. All children were able to comprehend the instructions and reliably report their own HRQoL. Completion of the questionnaire required 20 minutes.

\section{Statistical analysis}

The Statistical Package for the Social Sciences (SPSS version 16.0) was used to manage and analyze the data. First, missing values were handled according to the guidelines given in the manual of the KIDSCREEN-52. The percentage of missing data was $<10 \%$. Second, an independent $\mathrm{t}$-test and Chi square tests were used to compare children with SCD to healthy siblings on demographic characteristics. As demographic characteristics of the Dutch norm population of the KIDSCREEN-52 were not available, data on marital status, educational level, and employment in the general Dutch population was obtained from the Central Dutch Bureau of Statistics CBS http://www.cbs.nl. Third, linear mixed models were used to analyze differences in HRQoL between children with SCD and healthy siblings, and one-sample t-tests were used to analyze differences in HRQoL compared to the Dutch norm population of the KIDSCREEN-52. The linear mixed model allows for the investigation of group differences while controlling for the non-independency of data (i.e. more than one child participated per family, which resulted in related measurements within groups and between groups). All domains were analyzed using group (patients or healthy siblings) as fixed factor and family as random effect to account for within family correlation.

Besides analyzing the total study sample, we performed exploratory analyses separately for children aged 6-11 and adolescents aged 12-18 years, as the implications of SCD for children's social, emotional and cognitive development may vary depending on the impact of the disease at each stage of development [20]. Although the KIDSCREEN-52 was originally designed for children 8-18 years, we included 4 children aged 6 and 8 children aged 7 years. We explored differences in HRQoL between children aged 6-7 $(\mathrm{n}=12)$ and 8-18 years $(\mathrm{n}=$ 28) using independent $t$-tests, and performed all mixed model analyses with and without children aged 6-7 years. As no significant differences between these age groups were found, children aged 6-7 years were included in the final analyses.

Effect sizes (d) were calculated for differences between children with SCD and healthy siblings, by dividing the difference in mean score between children with SCD and healthy siblings by the pooled SD of both groups. According to Cohen [21], effect between 0.2 and 0.5 are considered small, effect sizes between 0.5 and 0.8 moderate, and effect sizes $>0.8$ large. Furthermore, we calculated the point estimate of the mean difference and confidence intervals between scores of children with SCD and the Dutch norm population [22].

To add clinical meaning, we evaluated how many children had impaired HRQoL scores. We followed Varni et al who defined impaired HRQoL scores as $\geq 1$ SD below the population mean [23]. The proportion of children with SCD with scores $\geq 1$ SD below the Dutch population mean was compared to the proportion of healthy siblings with scores $\geq 1$ SD below the Dutch population mean using Chi square test. Confidence intervals were calculated [22] for comparison of the proportion of children with SCD with impaired HRQoL to the proportion of children in the Dutch norm population. In the Dutch norm population, $16 \%$ of children have impaired HRQoL, based on the distribution of $\mathrm{T}$ values. A significance level of $p<0.05$ was used for all tests. Considering the exploratory nature of our study, we did not correct for multiple comparisons.

\section{Results}

\section{Demographics}

Table 1 provides the demographic characteristics of children with SCD, healthy siblings, and the general Dutch population. Children with SCD did not differ 
Table 1 Demographic characteristics of children with SCD, healthy siblings and the general Dutch population

\begin{tabular}{|c|c|c|c|c|c|c|}
\hline \multirow[b]{2}{*}{ Age in years, $M(S D)^{a}$} & \multicolumn{2}{|c|}{$\begin{array}{l}\text { Children with SCD } \\
\quad(n=40)\end{array}$} & \multicolumn{2}{|c|}{$\begin{array}{l}\text { Healthy siblings } \\
\quad(\mathrm{n}=36)\end{array}$} & \multicolumn{2}{|c|}{ General population $^{c}$} \\
\hline & 11.7 & (3.1) & 11.6 & (3.4) & - & - \\
\hline Boys, $n(\%)^{a}$ & 20 & (50) & 18 & (50) & - & - \\
\hline \multicolumn{7}{|l|}{ Country of origin ${ }^{a}$} \\
\hline Surinam, $n(\%)$ & 18 & (45) & 23 & (64) & - & - \\
\hline West/Central Africa, $n$ (\%) & 19 & (48) & 10 & (28) & - & - \\
\hline Turkey, n (\%) & 2 & (5) & 2 & (5) & - & - \\
\hline Netherlands Antilles, $n$ (\%) & 1 & (2) & 1 & (3) & - & - \\
\hline Parental marital status ${ }^{a}$ & & & & & $(\mathrm{n}=16.48$ & \\
\hline Married/living together, $n(\%)$ & 15 & (38) & 17 & (47) & 2.051 & $(81)$ \\
\hline Single, $n(\%)$ & 25 & (62) & 19 & (53) & 474 & (19) \\
\hline Highest level of education of mother ${ }^{a} b$ & & & & & $(n=2.03$ & \\
\hline Lower, n (\%) & 22 & (55) & 19 & (53) & 431 & (21) \\
\hline Intermediate, $n(\%)$ & 6 & (15) & 10 & (28) & 955 & (47) \\
\hline Higher, $n(\%)$ & 2 & (5) & 1 & (3) & 641 & (31) \\
\hline Not specified, $n(\%)$ & 10 & (25) & 6 & (17) & 13 & (1) \\
\hline Parental paid employment ${ }^{a}$ & & & & & $(n=3.92$ & \\
\hline Yes, $n(\%)$ & 22 & (55) & 23 & (64) & 3.787 & (96) \\
\hline No, $n(\%)$ & 16 & (40) & 9 & (25) & 141 & (4) \\
\hline Not specified, $n(\%)$ & 2 & (5) & 4 & (10) & - & - \\
\hline
\end{tabular}

${ }^{a}$ Non-significant difference between children with SCD and healthy siblings.

b Education: Lower: elementary education, general secondary education junior-level, lower vocational education; Intermediate: general secondary educationsenior level, and vocational education-junior level; Higher: vocational education-senior level and university education.

c Data obtained from the Central Dutch Bureau of Statistics CBS. All numbers $\times 10^{3}$.

${ }^{\mathrm{d}}$ Total Dutch population.

e Total number of Dutch females aged 25-45.

${ }^{f}$ Total number of Dutch people aged 25-45 who are part of the labour force.

significantly from healthy siblings in the distribution of age, gender, country of origin, parental marital status, maternal educational level or parental paid employment. However, compared to the general Dutch population, more children with SCD and healthy siblings grow up in single-parent families with lower educational levels and fewer paid employment.

\section{HRQoL of total study sample}

Results are reported in Table 2. Compared to healthy siblings, children with SCD had significantly lower HRQoL on only 1 domain of the KIDSCREEN-52: Physical Well-being. The effect size for this domain was moderate. However, compared to the Dutch norm population, children with SCD scored significantly lower on 5 domains: Physical Well-being, Moods \& Emotions, Autonomy, Parent Relation, and Financial Resources. Healthy siblings also scored significantly lower on Moods \& Emotions, Parent Relation, and Financial Resources compared to the Dutch norm population.

\section{HRQoL of children aged 6-11}

Results are reported in Table 3. Although children with SCD aged 6-11 years had slightly lower mean scores than healthy siblings on 8 of the 10 domains of the
KIDSCREEN-52, no statistically significant differences were found. There was a moderate effect size for lower HRQoL of children with SCD on Physical Well-being, compared to healthy siblings. Effect sizes for the other domains were small.

Compared to the Dutch norm population, children with SCD aged 6-11 years had significantly lower HRQoL on 7 of the 10 domains: Physical Well-being, Moods \& Emotions, Self-Perception, Autonomy, Parent Relation, Financial Resources, and Bullying. Healthy siblings had significantly lower mean scores on 3 of these domains: Autonomy, Parent Relation, and Financial Resources.

\section{HRQoL of children aged 12-18}

Results are reported in Table 4. Adolescents with SCD had a significantly lower HRQoL on Autonomy compared to healthy siblings. The effect size for this domain was moderate. Adolescents with SCD also had significantly lower scores on Autonomy compared to the Dutch norm population. No other significant differences compared to the Dutch norm population were found. Healthy siblings aged 12-18 years did no differ significantly from the Dutch norm population either, except for significantly lower scores on Moods \& Emotions. 
Table 2 HRQoL of total study sample of children with SCD compared to healthy siblings and Dutch norm population of the KIDSCREEN-52

\begin{tabular}{|c|c|c|c|c|c|c|c|c|}
\hline & \multicolumn{2}{|c|}{$\begin{array}{c}\text { Children } \\
\text { with SCD } \\
(n=40)\end{array}$} & \multicolumn{2}{|c|}{$\begin{array}{l}\text { Healthy } \\
\text { siblings } \\
(\mathrm{n}=36)\end{array}$} & \multirow{2}{*}{$\begin{array}{c}\text { SCD versus healthy siblings } \\
\text { Effect size }\end{array}$} & \multicolumn{2}{|c|}{$\begin{array}{c}\text { Norm } \\
\text { population } \\
(n=1960)\end{array}$} & \multirow[b]{2}{*}{ Point estimate of mean difference $(95 \% \mathrm{Cl})$} \\
\hline & Mean & SD & Mean & SD & & Mean & SD & \\
\hline Physical Well-being & $49 t^{*}$ & 8.7 & 54 & 11.4 & 0.5 & 53 & 10.0 & $-4(-7 ;-1)$ \\
\hline Psychological Well-being & 53 & 9.3 & 55 & 9.6 & 0.2 & 53 & 8.8 & $0(-3 ;+3)$ \\
\hline Moods \& Emotions & $47 *$ & 8.9 & $47 *$ & 10.2 & 0 & 51 & 9.6 & $-4(-7 ;-1)$ \\
\hline Self-Perception & 52 & 8.8 & 53 & 10.8 & 0.1 & 52 & 10.1 & $0(-3 ;+3)$ \\
\hline Autonomy & $48 *$ & 8.1 & 52 & 9.4 & 0.5 & 55 & 9.1 & $-7(-10 ;-4)$ \\
\hline Parent Relation & $50 *$ & 10.3 & $50 *$ & 10.4 & 0 & 53 & 9.1 & $-3(-6 ; 0)$ \\
\hline Financial Resources & $47 *$ & 10.0 & $46 *$ & 11.8 & 0.1 & 52 & 9.7 & $-5(-8 ;-2)$ \\
\hline Social Support \& Peers & 50 & 11.3 & 51 & 12.4 & 0.1 & 52 & 9.3 & $-2(-5 ; 0)$ \\
\hline School Environment & 54 & 8.6 & 54 & 10.0 & 0 & 53 & 10.0 & $1(-2 ;+4)$ \\
\hline Bullying & 45 & 11.5 & 47 & 11.5 & 0.6 & 49 & 10.4 & $-4(-7 ;-1)$ \\
\hline
\end{tabular}

Higher scores represent better HRQoL.

$+\mathrm{p}<0.05$ children with SCD versus healthy siblings.

${ }^{*} \mathrm{p}<0.05$ children with SCD and/or healthy siblings versus Dutch norm population of the KIDSCREEN-52.

\section{Proportion of children with impaired HRQoL}

Results are reported in Figure 1. The proportion of children with SCD with impaired HRQoL ( $\geq 1$ SD below the mean) was similar to the proportion of healthy siblings with impaired HRQoL. However, the proportion of children with SCD with impaired HRQoL was significantly larger than the proportion in the Dutch norm population on Physical Well-being, Moods \& Emotions, Autonomy, Parent Relation, Financial Resources, and Bullying. More than 1 in 3 children with SCD (between $30-38 \%$ ) had impaired HRQoL on these domains, which is a twofold increase in comparison to the Dutch norm population (16\%). The proportion of healthy siblings with impaired HRQoL was also significantly larger than the proportion in the Dutch norm population on
Moods \& Emotions, Autonomy, Parent Relation, and Financial Resources.

\section{Discussion}

This is the first study to assess multidimensional HRQoL in children with SCD using a self-report measure and a control group of healthy siblings. In general, the HRQoL of children with SCD appeared similar to the HRQoL of healthy siblings, with the exception of lower scores on the physical domain for children with $\mathrm{SCD}$, and, specifically in adolescents, lower scores on the autonomy domain. However, the HRQoL of both children with SCD and healthy siblings was considerably lower than the Dutch norm population on several domains, specifically in children aged 6-11. This implies

Table 3 HRQoL of children with SCD aged 6-11 compared to age matched healthy siblings and Dutch norm population of the KIDSCREEN-52

\begin{tabular}{lllllcccc}
\hline & $\begin{array}{c}\text { Children } \\
\text { with SCD } \\
\text { (n= 17) }\end{array}$ & \multicolumn{2}{c}{$\begin{array}{c}\text { Healthy } \\
\text { siblings } \\
\text { (n= 19) }\end{array}$} & SCD versus healthy siblings & $\begin{array}{c}\text { Norm } \\
\text { population } \\
\text { (n= 641) }\end{array}$ & SCD versus norm population \\
\hline & Mean & SD & Mean & SD & Effect size & Mean SD & Point estimate of mean difference (95\% CI) \\
\hline Physical Well-being & $51 *$ & 9.5 & 58 & 11.7 & 0.7 & 57 & 9.5 & $-6(-11 ;+1)$ \\
Psychological Well-being & 55 & 8.7 & 57 & 9.1 & 0.2 & 56 & 9.1 & $-1(-5 ;+3)$ \\
Moods \& Emotions & $46 *$ & 11.0 & 48 & 12.3 & 0.2 & 53 & 9.5 & $-7(-12 ;-2)$ \\
Self-Perception & $50 *$ & 7.9 & 54 & 11.0 & 0.4 & 57 & 9.8 & $-7(-12 ;-2)$ \\
Autonomy & $49 *$ & 6.8 & $50 *$ & 9.4 & 0.1 & 57 & 8.6 & $-8(-12 ;-4)$ \\
Parent Relation & $48 *$ & 10.4 & $50 *$ & 10.1 & 0.2 & 56 & 8.4 & $-8(-12 ;-4)$ \\
Financial Resources & $44 *$ & 11.8 & $43 *$ & 14.2 & 0.1 & 51 & 10.6 & $-7(-12 ;-2)$ \\
Social Support \& Peers & 53 & 11.7 & 49 & 13.9 & 0.3 & 53 & 9.1 & $0(-4 ;+4)$ \\
School Environment & 56 & 8.1 & 57 & 11.2 & 0.1 & 58 & 10.2 & $-2(-7 ;+3)$ \\
Bullying & $42 *$ & 10.9 & 46 & 12.6 & 0.3 & 48 & 11.0 & $-6(-11 ;+1)$ \\
\hline
\end{tabular}

Higher scores represent better HRQoL.

${ }^{*} p<0.05$ children with SCD and/or healthy siblings versus Dutch norm population of the KIDSCREEN-52. 
Table 4 HRQoL of adolescents with SCD aged 12-18 compared to age matched healthy siblings and Dutch norm population of the KIDSCREEN-52

\begin{tabular}{|c|c|c|c|c|c|c|c|c|}
\hline & \multicolumn{2}{|c|}{$\begin{array}{c}\text { Children } \\
\text { with SCD } \\
(\mathrm{n}=23)\end{array}$} & \multicolumn{2}{|c|}{$\begin{array}{l}\text { Healthy } \\
\text { siblings } \\
(n=17)\end{array}$} & \multirow{2}{*}{$\begin{array}{c}\text { SCD versus healthy siblings } \\
\text { Effect size }\end{array}$} & \multicolumn{2}{|c|}{$\begin{array}{c}\text { Norm } \\
\text { population } \\
(n=1270)\end{array}$} & \multirow[b]{2}{*}{ Point estimate of mean difference $(95 \% \mathrm{Cl})$} \\
\hline & Mean & SD & Mean & SD & & Mean & SD & \\
\hline Physical Well-being & 48 & 8.0 & 50 & 9.5 & 0.2 & 50 & 9.4 & $-2(-6 ;+2)$ \\
\hline Psychological Well-being & 51 & 9.5 & 53 & 9.7 & 0.2 & 52 & 8.4 & $-1(-5 ;+3)$ \\
\hline Moods \& Emotions & 48 & 7.2 & $46 *$ & 7.4 & 0.3 & 51 & 9.6 & $-3(-7 ;+1)$ \\
\hline Self-Perception & 54 & 9.2 & 52 & 10.9 & 0.2 & 50 & 9.3 & $4(0 ;+8)$ \\
\hline Autonomy & $47 \dagger^{*}$ & 9.0 & 53 & 9.5 & 0.7 & 54 & 9.1 & $-7(-11 ;-3)$ \\
\hline Parent Relation & 51 & 10.2 & 49 & 11.0 & 0.2 & 52 & 9.3 & $-1(-5 ;+3)$ \\
\hline Financial Resources & 50 & 7.8 & 50 & 7.0 & 0 & 53 & 9.2 & $-3(-7 ;+1)$ \\
\hline Social Support \& Peers & 48 & 10.8 & 53 & 10.4 & 0.5 & 52 & 9.4 & $-4(-8 ; 0)$ \\
\hline School Environment & 53 & 8.8 & 49 & 6.4 & 0.5 & 51 & 8.7 & $2(-2 ;+6)$ \\
\hline Bullying & 48 & 11.6 & 49 & 10.5 & 0.1 & 49 & 10.1 & $-1(-5 ;+3)$ \\
\hline
\end{tabular}

Higher scores represent better HRQoL.

$+\mathrm{p}<0.05$ children with SCD versus healthy siblings.

${ }^{*} p<0.05$ children with SCD and/or healthy siblings versus Dutch norm population of the KIDSCREEN-52.

that reduced HRQoL in children with SCD is primarily related to the low SES of this patient population, although there are disease specific effects on the physical and autonomy domain.

A large study in urban elementary schoolchildren from poor socioeconomic areas formerly established the essential impact that SES can have on HRQoL. These children reported lower HRQoL than chronically diseased children [24]. Nevertheless, prior studies in children with SCD suggested that the HRQoL of children with SCD is impaired, even after considering the potential detrimental effect of low SES [15-17]. The contrast between these previous findings and the present results may be explained by the use of different instruments, different comparison groups and particularly the use of proxy- versus self-report. In our study, a self-report questionnaire was administered, while other researchers have mainly used parental proxy-report. Parents of chronically diseased children generally tend to report worse HRQoL for their children than children themselves $[25,26]$, which was confirmed by previous studies in children with SCD $[13,16,17]$. This may have led to an underestimation of the HRQoL of children with SCD. Parents could underestimate their children's HRQoL as a result of parental distress [27]. Alternatively, parents and children could have different perceptions: while children may be unaware of the potential consequences of the disease, parents may have greater concerns for their future [28] and take on a protective attitude.

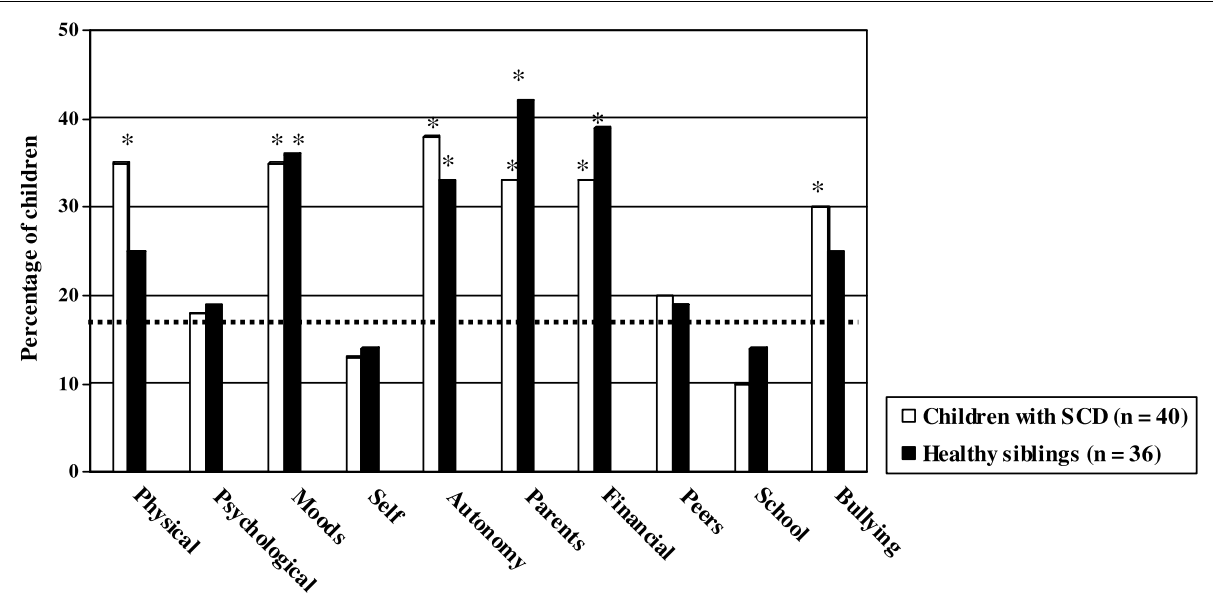

Figure 1 Proportions of children with SCD and healthy siblings with impaired HRQoL ( $\geq 1$ SD below the mean of the Dutch norm population of the KIDSCREEN-52). The dotted line represents the $16 \%$ of children in the Dutch norm population of the KIDSCREEN- 52 with impaired HRQoL. * 95\% Confidence interval of the proportion of children with SCD or healthy siblings with impaired HRQoL exceeds the proportion in the Dutch norm population. 
Parental (over-)protection could also underlie our finding of lower scores of adolescents with SCD on the autonomy domain, compared to healthy siblings. This finding is congruent with results of a previous study in our hospital [13] as well as other studies on adjustment in adolescents with SCD $[29,30]$, and seems to be associated with consequences of the disease. Therefore, we recommend that parental overprotection should be considered and targeted in intervention programs for adolescents with SCD. As higher levels of self-efficacy were previously found to be associated with fewer SCD symptoms [30], interventions to increase autonomy could possibly lead to a decrease in SCD symptomatology in this age group.

Interestingly, adolescents did not differ significantly from healthy siblings or the Dutch norm population on any of the other domains except for autonomy, while children aged 6-11 did have significantly lower scores than the norm population on 7 of the 10 domains. Although adolescents have been described to generally experience more problems in psychosocial adaptation than younger children [7], HRQoL scores of adolescents were within the normative range in a previous study as well [27]. The resilience of adolescents could be a consequence of developmental growth and adjustment, possibly causing a better coping style with increasing age. This should be investigated further longitudinally.

Surprisingly, children with SCD did not report any social problems. This is a remarkable contrast to the results of previous findings [6-10], including results of our own work in which parents rated their children with SCD to show less competent social behavior than healthy siblings [31]. Furthermore, it is striking that children with SCD did not report a lower HRQoL on the School environment domain, as studies on neurocognitive sequelae of SCD have shown a decrease in general intellectual ability as well as deficits in specific neurocognitive domains in children with SCD [32]. Children with SCD may have adjusted to their social and cognitive deficits and therefore do not subjectively experience HRQoL problems in these domains. In future research behavioural and neurocognitive data should be correlated to HRQoL, to gain more insight into the relation between objectively identified problems and the subjective HRQoL of children with SCD.

While interpreting the results of this study, strengths and limitations should be taken into account. Findings of this study are strengthened by the use of a well-standardized self-report measure and robust statistical methods to take within family correlations into account. Furthermore, by including a control group of healthy siblings with the same age, ethnicity and SES, the differential effect of low SES and disease specific consequences of SCD on HRQoL could be determined.
However, not all participating children with SCD had an eligible sibling, causing us to recruit healthy siblings from the entire SCD patient population. This led to an overrepresentation of children from Surinam descent in the healthy sibling group. Nevertheless, demographic differences between the groups were not statistically significant and explorative within-group analyses revealed no significant differences between children from Surinam or African descent on any of the HRQoL domains. Another limitation was the small sample size, mitigating statistical power. The results of our exploratory analyses need to be interpreted within this limitation. Moreover, the current study design did not allow us to investigate if there is an interaction effect of SES and sickle cell disease on HRQoL. Further limitations concerned the instrument we used to assess HRQoL. The KIDSCREEN-52 is not yet validated in children aged 6 and 7 . However, young participants received cognitive debriefing to ascertain reliable self-report, and this issue was taken into account in statistical analyses. Children as young as 5 years of age have been found to be able to reliably report HRQoL $[26,33,34]$. Furthermore, data were collected in a clinical setting, which may have affected the response of the participants. Finally, the KIDSCREEN-52 assesses HRQoL of the past week. As SCD has a very unpredictable course, it would be more appropriate to assess HRQoL over longer time periods, e.g. the past month. These issues should be taken into account in future research.

\section{Conclusion}

Based on the present findings, we conclude that children with SCD are primarily disadvantaged by their low SES, causing lower HRQoL on several domains compared to the Dutch norm population. Nevertheless, they are also affected by specific consequences of the disease, reflected by the lower HRQoL on the physical and autonomy domains compared to healthy siblings. Moreover, one in three children with SCD experience impaired HRQoL on several domains. As children with SCD are not only affected by their disease but also by their low SES, children with SCD seem to be especially vulnerable compared to other patient populations, and need specific care in the hospital. Therefore, we argue for routine monitoring of HRQoL in children with SCD. Incorporating patient reported outcomes of HRQoL in daily clinical practice will contribute to better communication with health care professionals. This can provide these children with the additional care they need due to their double disadvantage: a chronic disease, on top of an unfavourable background.

\section{Declaration of competing interests}

The authors declare that they have no competing interests. 


\section{Acknowledgements}

The authors would like to thank all the participating children for their willingness to participate in this study. This work was supported by AGIS health care.

\section{Author details}

'Psychosocial Department, Emma Children's Hospital, Academic Medical Center, P.O. Box 304, 1100 VC Amsterdam, The Netherlands. ${ }^{2}$ Department of Pediatric Hematology, Emma Children's Hospital, Academic Medical Center, P.O. Box 304, 1100 VC Amsterdam, The Netherlands. ${ }^{3}$ Department of Clinical Neuropsychology, VU University Amsterdam, Van der Boechorststraat 1, 1081 BT Amsterdam, The Netherlands.

\section{Authors' contributions}

$\mathrm{CTH}$ collected the data, analyzed and interpreted the data and drafted the manuscript. KF designed and supervised execution of the study, interpreted the data and drafted and revised the manuscript. $\mathrm{JO}, \mathrm{HH}$ and MP supervised execution of the study and revised the manuscript. MAG designed and supervised execution of the study, interpreted the data and drafted and revised the manuscript. All authors read and approved the manuscript.

Received: 31 May 2010 Accepted: 26 October 2010

Published: 26 October 2010

\section{References}

1. Stuart MJ, Nagel RL: Sickle-cell disease. Lancet 2004, 364:1343-1360.

2. Roberts I, de Montalembert M: Sickle cell disease as a paradigm of immigration hematology: new challenges for hematologists in Europe. Haematologica 2007, 92:865-871.

3. Van den Tweel $\mathrm{X}$, Hatzmann J. Ensink E, van der Lee JH, Peters $\mathrm{M}$ Fijnvandraat K, et al: Quality of life of female caregivers of children with sickle cell disease: a survey. Haematologica 2008, 93:588-593.

4. World Health Organization: Preamble to the Constitution of the World Health Organization as adopted by the International Health Conference, New York, 19-22 June 1946. New York 1946.

5. Berra S, Ravens-Sieberer U, Erhart M, Tebe C, Bisegger C, Duer W, et al: Methods and representativeness of a European survey in children and adolescents: the KIDSCREEN study. BMC Public Health 2007, 7:182.

6. Anie KA: Psychological complications in sickle cell disease. Br J Haematol 2005, 129:723-729.

7. Barakat L, Lash LA, Lutz MJ, Nicolaou DC: Psychosocial Adaptation of Children and Adolescents with Sickle Cell Disease. In Comprehensive Handbook of Childhood Cancer and Sickle Cell Disease. Edited by: Brown RT. Oxford University Press; 2006:471-495.

8. Noll RB, Vannatta K, Koontz K, Kalinyak K, Bukowski WM, Davies WH: Peer relationships and emotional well-being of youngsters with sickle cell disease. Child Dev 1996, 67:423-436.

9. Noll RB, Reiter-Purtill J, Vannatta K, Gerhardt CA, Short A: Peer relationships and emotional well-being of children with sickle cell disease: a controlled replication. Child Neuropsychol 2007, 13:173-187.

10. Trzepacz AM, Vannatta K, Gerhardt CA, Ramey C, Noll RB: Emotional, social, and behavioral functioning of children with sickle cell disease and comparison peers. Journal of Pediatric Hematology/Oncology 2004, 26:642-648.

11. Panepinto JA: Health-related quality of life in sickle cell disease. Pediatr Blood Cancer 2008, 51:5-9.

12. Bonner MJ: Health related quality of life in sickle cell disease: just scratching the surface. Pediatr Blood Cancer 2010, 54:1-2.

13. Kater AP, Heijboer H, Peters M, Vogels T, Prins MH, Heymans HSA: Quality of life of children with sickle cell disease in the Amsterdam area. Nederlands Tijdschrift voor Geneeskunde 1999, 143:2049-2053.

14. Palermo TM, Schwartz L, Drotar D, McGowan K: Parental report of healthrelated quality of life in children with sickle cell disease. J Behav Med 2002, 25:269-283.

15. Palermo TM, Riley CA, Mitchell BA: Daily functioning and quality of life in children with sickle cell disease pain: relationship with family and neighborhood socioeconomic distress. J Pain 2008, 9:833-840.

16. Panepinto JA, O'Mahar KM, DeBaun MR, Loberiza FR, Scott JP: Healthrelated quality of life in children with sickle cell disease: child and parent perception. Br J Haematol 2005, 130:437-444.
17. Panepinto JA, Pajewski NM, Foerster LM, Sabnis S, Hoffmann RG: Impact of family income and sickle cell disease on the health-related quality of life of children. Qual Life Res 2009, 18:5-13.

18. McClellan CB, Schatz J, Sanchez C, Roberts CW: Validity of the Pediatric Quality of Life Inventory for Youth with Sickle Cell Disease. J Pediatr Psychol 2008, 33:1153-1162.

19. Ravens-Sieberer U, Gosch A, Rajmil L, Erhart M, Bruil J, Power M, et al: The KIDSCREEN-52 quality of life measure for children and adolescents: psychometric results from a cross-cultural survey in 13 European countries. Value Health 2008, 11:645-658.

20. Gustafson KE, Bonner MJ, Hardy KK, Thompson RJ: Biopsychosocial and Developmental Isses in Sickle Cell Disease. In Comprehensive Handbook of Childhood Cancer and Sickle Cell Disease. Edited by: Brown RT. New York: Oxford University Press; 2006:431-448.

21. Cohen J: Statistical power analysis for the behavioral sciences New York: Academy Press; 1988.

22. Gardner MJ, Altman DG: Statistics with confidence London: British Medical Journal; 1989.

23. Varni JW, Burwinkle TM, Seid M, Skarr D: The PedsQL 4.0 as a pediatric population health measure: feasibility, reliability, and validity. Ambul Pediatr 2003, 3:329-341.

24. Mansour ME, Kotagal U, Rose B, Ho M, Brewer D, Roy-Chaudhury A, et al: Health-related quality of life in urban elementary schoolchildren. Pediatrics 2003, 111:1372-1381.

25. Eiser C, Morse R: Can parents rate their child's health-related quality of life? Results of a systematic review. Qual Life Res 2001, 10:347-357.

26. Varni JW, Limbers CA, Burwinkle TM: Parent proxy-report of their children's health-related quality of life: An analysis of 13,878 parents' reliability and validity across age subgroups using the PedsQL 4.0 Generic Core Scales. Health and Quality of Life Outcomes 2007, 5:2.

27. Barakat LP, Patterson CA, Daniel LC, Dampier C: Quality of life among adolescents with sickle cell disease: mediation of pain by internalizing symptoms and parenting stress. Health Qual Life Outcomes 2008, 6:60.

28. Eiser C, Morse R: Quality-of-life measures in chronic diseases of childhood. Health Technol Assess 2001, 5:1-157.

29. Anie KA, Telfair J: Multi-site study of transition in adolescents with sickle cell disease in the United Kingdom and the United States. Int J Adolesc Med Health 2005, 17:169-178.

30. Clay OJ, Telfair J: Evaluation of a disease-specific self-efficacy instrument in adolescents with sickle cell disease and its relationship to adjustment. Child Neuropsychol 2007, 13:188-203.

31. Hijmans $C T$, Grootenhuis MA, Oosterlaan J, Last BF, Heijboer H, Peters M, et al: Behavioral and emotional problems in children with sickle cell disease and healthy siblings: Multiple informants, multiple measures. Pediatric Blood and Cancer 2009, 53:1277-1283.

32. Schatz J, Puffer E: Neuropsychological Aspects of Sickle Cell Disease. In Comprehensive Handbook of Childhood Cancer and Sickle Cell Disease. Edited by: Brown RT. New York Oxford University Press; 2006:449-470.

33. Feeny D, Furlong W, Boyle M, Torrance GW: Multi-attribute health status classification systems. Health Utilities Index. Pharmacoeconomics 1995, 7:490-502.

34. Juniper EF, Guyatt GH, Feeny DH, Griffith LE, Ferrie PJ: Minimum skills required by children to complete health-related quality of life instruments for asthma: comparison of measurement properties. Eur Respir J 1997, 10:2285-2294.

doi:10.1186/1477-7525-8-12

Cite this article as: Hijmans et al:: Double disadvantage: a case control study on health-related quality of life in children with sickle cell disease. Health and Quality of Life Outcomes 2010 8:121. 